\title{
Política fiscal e nível de atividade: breve resenha da literatura empírica e lições para o Brasil
}

\author{
Marco A. F. H. Cavalcanti*
}

RESUMO - Esta nota apresenta uma breve resenha da literatura empírica referente aos efeitos da política fiscal sobre o nível de atividade e discute as implicações dessa literatura para a condução da política fiscal no Brasil.

Palavras-chave: Política fiscal. Multiplicador fiscal. Políticas anticíclicas.

\section{INTRODUÇÃO}

A crise global de 2008-2009 reacendeu o interesse dos economistas e policymakers no uso da política fiscal como meio de atenuação dos ciclos econômicos. Em uma conjuntura na qual a política monetária se revelava ineficaz para combater os efeitos perversos da crise financeira sobre a economia real, a maioria dos países atingidos pela crise recorreu a diversos estímulos fiscais visando evitar quedas mais fortes no nível de atividade. Segundo estimativa da OCDE de março/2009, por exemplo, o tamanho dos pacotes de estímulo fiscal adotados para combater a crise nos países associados chegou, em média, a 2,5\% do PIB (OECD, 2009). No Brasil, as medidas anticíclicas de desoneração tributária e aumento de gastos públicos teriam atingido, segundo Pires (2009), R $\$ 43,4$ bilhões - ou cerca de 1,4\% do PIB.

Há, porém, grande incerteza entre os economistas quanto ao efeito da política fiscal sobre o nível de atividade. Conforme ressaltado por um dos especialistas nesse assunto, Roberto Perotti:

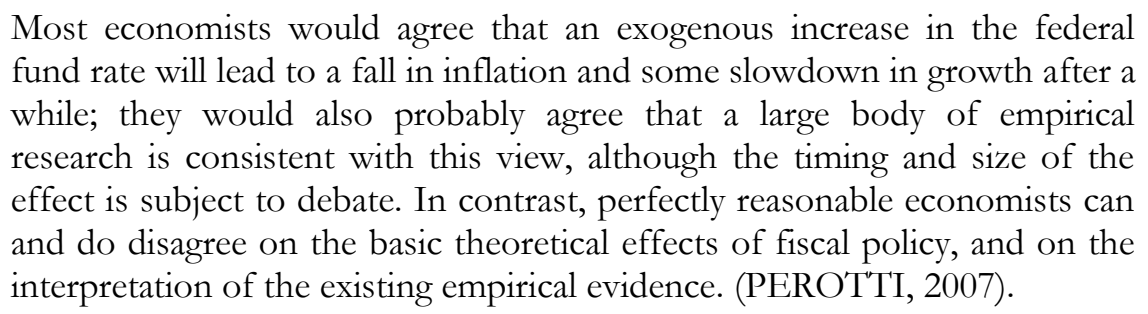

Tal incerteza deriva, de um lado, da multiplicidade de hipóteses teóricas acerca da relação entre política fiscal e nível de atividade; e, de outro lado, da dificuldade de se estimar adequadamente essa relação. Do ponto de vista teórico, podem-se distinguir pelo menos três

*IPEA e PUC-Rio. Endereço eletrônico: marco.cavalcanti@ipea.gov.br. 
visões alternativas da questão. Segundo a visão keynesiana tradicional, expansões fiscais caracterizadas por aumentos dos gastos públicos ou reduções de impostos - estimulam a economia, ao passo que contrações fiscais geram redução do nível de atividade. De acordo com uma segunda visão, a atuação da política fiscal através do canal da riqueza ou do canal das expectativas pode atenuar ou até mesmo reverter os efeitos keynesianos tradicionais, de modo que expansões fiscais podem gerar redução do produto, enquanto que contrações fiscais podem expandir a atividade econômica. Por fim, os adeptos da proposição de "Equivalência Ricardiana" defendem que, sob certas circunstâncias, expansões fiscais podem ser neutras no que se refere ao nível de atividade. ${ }^{1}$

Do ponto de vista empírico, a dificuldade de estimação dos efeitos da política fiscal sobre o produto está associada à dificuldade de diferenciar adequadamente o "componente exógeno" das variáveis fiscais (associado a mudanças discricionárias de política) de seu "componente endógeno" (associado ao ciclo econômico). Por exemplo, o aumento do déficit público observado pode ser devido a políticas discricionárias de elevação de gastos e/ou redução de impostos, ou pode ser o resultado de uma política expansionista que vise preservar o nível de atividade, face à expectativa de desaceleração econômica associada a algum outro evento macroeconômico observado pelas autoridades. Caso a expansão fiscal seja seguida por uma redução na taxa de crescimento do PIB, fica então a dúvida: a queda no crescimento do produto foi causada pela expansão fiscal, constituindo evidência favorável à hipótese de "efeitos não keynesianos da política fiscal"? Ou a redução no nível de atividade foi conseqüência de algum outro evento macroeconômico, sendo que, na verdade, a expansão fiscal atenuou a desaceleração econômica?

Dado que os efeitos da política fiscal sobre o nível de atividade são ambíguos, é natural que os economistas divirjam quanto à avaliação da eficácia e adequação de medidas fiscais "anticíclicas". A fim de contribuir para o debate, esta nota apresenta uma breve resenha da literatura empírica referente aos efeitos da política fiscal sobre o nível de atividade e discute as implicações dessa literatura para a condução da política fiscal no Brasil.

\section{LITERATURA INTERNACIONAL: PRINCIPAIS RESULTADOS}

Os efeitos da política fiscal sobre o nível de atividade podem ser estimados a partir de diferentes abordagens:

\footnotetext{
${ }^{1}$ Para discussões resumidas dessas visões, ver, dentre outros, Giavazzi et al. (2000), Hemming et al. (2002) e Perotti (2007).
}

16 
(i) Análise de "episódios de consolidação fiscal", como em Giavazzi e Pagano (1990) e Giavazzi et al.(2000): identificam-se situações em que ocorreram "ajustamentos fiscais significativos" (definidos de forma relativamente arbitrária - por exemplo, ajustamentos fiscais correspondentes a pelo menos 3\% do PIB ao longo de dois anos) e busca-se verificar se a resposta das variáveis reais a mudanças nas variáveis fiscais é diferente durante tais episódios relativamente a períodos "normais". Esse tipo de análise costuma basear-se no uso de dados em painel para amostras de países, em contraste com as abordagens listadas a seguir, que exploram predominantemente séries temporais para um país específico.

(ii) Abordagem da "variável dummy", como em Ramey e Shapiro (1998), Edelberg et al. (1999) e Burnside et al. (2004): identificam-se eventos fiscais "exógenos" associados, nos EUA, a gastos militares - e constrói-se uma variável dummy correspondente a tais eventos, que é então incorporada a um modelo autorregressivo vetorial (VAR) a fim de verificar seu efeito sobre o PIB, consumo etc. Dado que a dummy é, por definição, exógena, é suficiente considerar o VAR na forma reduzida, não sendo necessárias hipóteses de identificação adicionais.

(iii) Abordagem "narrativa" de Romer e Romer (2007), baseada na análise da legislação e documentos oficiais a fim de identificar o tamanho e motivação principal das políticas tributárias adotadas. Uma vez identificadas as mudanças tributárias consideradas "exógenas" (isto é, não motivadas pelo desejo de fazer o produto voltar a seu nível "normal”), estima-se uma regressão da taxa de crescimento do PIB nessa medida de "choques fiscais".

(iv) Abordagem do "VAR Estrutural" (SVAR), baseada na identificação dos choques fiscais "estruturais" a partir de informações sobre o "timing" e outras características institucionais da política fiscal, como em Fatás e Mihov (2001), Blanchard e Perotti (2002) e Perotti (2005), ou a partir de hipóteses sobre os sinais das respostas esperadas a choques reais, monetários e fiscais, como em Mountford e Uhlig (2005). A maioria dos estudos nessa linha refere-se a análises de séries temporais, mas vale destacar o estudo recente de Ilzetzki et al.(2009), que aplica o método a um painel de 45 países.

(v) Simulações a partir de modelos macroeconômicos calibrados ou estimados diretamente na forma "estrutural", tais como em Adams e Klein (1991), que usam modelos macroeconométricos à la Cowles Commission, ou em Cogan et al.(2009) 
e Forni et al.(2009), que adotam modelos dinâmicos estocásticos de equilíbrio geral (DSGE).

As estimativas dos "multiplicadores fiscais" podem variar significativamente em função da abordagem adotada, bem como de características dos modelos estimados dentro de cada abordagem (variáveis de controle utilizadas, método de identificação dos choques fiscais, regra de política monetária implícita, período amostral etc.). Resenhas detalhadas da literatura empírica são apresentadas em Hemming et al.(2002), Van Brusselen (2009), Spilimbergo (2009) e OECD (2009); a seguir, listam-se apenas os resultados que parecem razoavelmente robustos ao método de estimação utilizado:

- Em países desenvolvidos, o multiplicador fiscal é quase sempre positivo, seja no curto prazo (1 ano) como no médio prazo (2 ou 3 anos).

- Em países em desenvolvimento, a resposta do produto a um aumento nos gastos do governo é menor e menos persistente do que em países desenvolvidos - havendo, assim, maior evidência de "crowding out" de gastos privados.

- A magnitude do multiplicador varia com o tipo de medida fiscal: os maiores multiplicadores estão associados a gastos de investimento do governo, seguidos por gastos de consumo e transferências às famílias. Os menores multiplicadores são aqueles relativos às mudanças tributárias.

- Quanto maior o grau de abertura da economia, menor tende a ser o multiplicador fiscal - pois é mais provável que parte do aumento de demanda agregada seja absorvido por importações.

- Quanto maior a flexibilidade do regime cambial, menor é a magnitude do multiplicador fiscal - em conformidade com modelos macroeconômicos padrão à la Mundell-Fleming.

- A magnitude do multiplicador fiscal tende a ser menor em países com elevado endividamento externo, pois, nesse contexto, políticas fiscais expansionistas podem resultar em maiores déficits em conta corrente, causando aumento do risco-país e das taxas de juros - e, consequentemente, redução do nível de atividade.

- Em países com elevada razão dívida pública/PIB, os multiplicadores fiscais tendem a ser menores do que em países em que não há "restrição fiscal", podendo ocorrer "efeitos não-keynesianos" da política fiscal - isto é, contrações fiscais expansionistas ou expansões fiscais contracionistas. 


\section{RESULTADOS PARA O BRASIL}

Os estudos de multiplicadores fiscais para o Brasil são escassos, ${ }^{2}$ e os resultados ambíguos. A análise de Peres (2006), baseada na metodologia VAR de Blanchard e Perotti (2002) para o período 1994-2005, dá sustentação à visão keynesiana tradicional, no sentido de que elevações inesperadas nos gastos públicos (federais) estão positivamente relacionadas com o produto e elevações na carga tributária líquida estão negativamente relacionadas com o produto. Esse trabalho parece confirmar resultados anteriores obtidos por Reis et al.(1999) para o período 1960-1997, no contexto de um modelo macroeconométrico de larga escala à la Cowles Commission.

Outros estudos, porém, apresentam resultados compatíveis com a ocorrência de “efeitos não-keynesianos" da política fiscal. Mendonça et al. (2009), baseados na abordagem de Mountford e Uhlig (2005), concluem que um aumento inesperado do gasto corrente do governo pode conduzir, com elevada probabilidade, a uma retração do produto real, enquanto que um choque positivo na carga tributária líquida pode levar, a médio prazo, a uma resposta positiva do PIB.

Cavalcanti e Silva (2009) seguem a recomendação de Favero e Giavazzi (2007) e estimam os efeitos da política fiscal na economia brasileira no período 1995-2008 a partir de uma análise VAR que leva explicitamente em consideração o papel da dívida pública na determinação da política fiscal. Os resultados obtidos também parecem sustentar a hipótese de efeitos "não-keynesianos" da política fiscal, indicando efeito próximo de zero dos gastos públicos sobre o PIB e efeito levemente positivo, a médio prazo, de aumentos da receita tributária sobre a atividade. A interpretação é que, na maior parte do período analisado, o país esteve sob restrição fiscal e havia grande preocupação com a solvência da dívida pública; logo, choques fiscais que causassem o aumento da relação dívida/PIB deviam acarretar uma resposta de política compensatória, reduzindo gastos ou aumentando impostos, de modo a manter a dívida sob controle - o que implicava reduzido efeito dos choques sobre o nível de atividade.

Silva e Cândido Júnior (2009) mensuram os impactos macroeconômicos dos gastos públicos nas principais economias da América Latina, inclusive no Brasil, a partir de modelos VAR cointegrados estimados separadamente para cada país no período 1970-2002. Os

\footnotetext{
${ }^{2}$ Cabe ressaltar que nosso foco é nos estudos dos efeitos da política fiscal no curto e médio prazo. Não se consideram, assim, trabalhos que analisam o possível impacto dos gastos públicos sobre o crescimento de longo prazo da economia brasileira. A esse respeito, ver, dentre outros, Ferreira (1994, 1996).
} 
resultados para o Brasil indicam multiplicadores fiscais não significativamente diferentes de zero no curto prazo, e relações negativas entre gastos públicos e privados no longo prazo também apontando, portanto, para a ocorrência de efeitos não-keynesianos da política fiscal.

\section{LIÇÕES PARA A CONDUÇÃO DA POLÍTICA FISCAL NO BRASIL}

Os resultados da literatura empírica internacional deixam claro que o sinal e magnitude da resposta do PIB a medidas de política fiscal variam em função das características e especificidades de cada país. No que se refere ao Brasil, as implicações dessa literatura são ambíguas: de um lado, o fato do país ser relativamente fechado ao comércio externo aponta para a ocorrência de multiplicadores fiscais positivos e elevados; de outro lado, a flexibilidade do regime cambial brasileiro e a magnitude ainda elevada da razão dívida pública/PIB sugerem que os multiplicadores fiscais podem não ser tão significativos.

Esses efeitos contraditórios podem explicar parcialmente os resultados ambíguos dos estudos recentes para o Brasil. Em particular, parece razoável que no período pós-Real, e principalmente a partir de 1999, a combinação de maior flexibilidade cambial com dívida pública elevada e forte restrição fiscal tenha atuado no sentido de gerar multiplicadores fiscais insignificantes ou até negativos - em conformidade, portanto, com as estimativas de Mendonça et al.(2009) e Cavalcanti e Silva (2009).

Vale notar, porém, que nos últimos anos a relação dívida/PIB vinha caindo sistematicamente, afetando positivamente a percepção de sustentabilidade da política fiscal brasileira, pelo menos a médio prazo. Assim, quando a crise global se abateu sobre o Brasil em 2008, é provável que a política fiscal já tivesse recuperado, pelo menos parcialmente, a capacidade de atuar como instrumento de estabilização da economia. Aliado ao caráter e profundidade da crise, que implicaram a possibilidade de adoção simultânea de medidas de estímulo monetário e fiscal sem pressão sobre a inflação e os juros, isso permite supor que a política fiscal expansionista adotada no país a partir do final de 2008 tenha realmente contribuído para atenuar os efeitos adversos da crise sobre o nível de atividade econômica.

Isso não significa, porém, que as medidas adotadas tenham sido as medidas ideais. De acordo com a literatura internacional, os multiplicadores fiscais de curto e médio prazo são maiores para gastos de investimento, que ainda apresentam a vantagem de poder contribuir para o aumento da produtividade e crescimento de longo prazo - notadamente, quando se trata de gastos em infra-estrutura -; entretanto, a maior parte dos estímulos fiscais adotados no país esteve associada a isenções fiscais e gastos correntes. Nesse sentido, a 
composição do pacote de estímulos fiscais poderia ter sido melhor. Evidentemente, deve-se ter em mente que a implementação de um programa de investimentos públicos requer tempo; dada a rapidez com que a economia brasileira foi atingida pela crise em fins de 2008, talvez não houvesse alternativas viáveis a algumas das medidas efetivamente adotadas - em especial, as desonerações tributárias, rapidamente implementáveis e facilmente reversíveis no futuro. Entretanto, a forte ênfase no aumento de gastos de pessoal e custeio foi, no mínimo, questionável, dado o elevado custo de oportunidade dos recursos públicos no meio da crise.

No momento atual, em que o pior da crise ficou para trás e a demanda agregada volta a crescer a taxas elevadas, parece chegada a hora de reverter os estímulos fiscais. De fato, diante do reaquecimento da economia, a manutenção de uma política fiscal expansionista pode contribuir para o aumento da inflação, levando o Banco Central a elevar a taxa de juros e, assim, causando crowding-out de investimentos privados - com conseqüências perversas para o potencial de crescimento da economia em prazo mais longo. Além disso, a expansão fiscal também pode exacerbar a tendência atual ao aumento do déficit em conta corrente do balanço de pagamentos, recolocando a economia em uma arriscada posição de dependência em relação aos influxos de capital externo.

Olhando para frente, a possibilidade de se continuar, no futuro, a usar políticas fiscais anticíclicas na estabilização dos ciclos econômicos depende crucialmente da manutenção da divida pública em níveis sustentáveis. Dados os efeitos adversos da crise sobre o PIB e as receitas tributárias, bem como a resposta de política econômica baseada em desonerações tributárias e ampliação de gastos, verificou-se em 2009 uma inflexão não desprezível na trajetória de queda da razão dívida/PIB iniciada em 2002. Ainda não parece haver motivo para preocupações sobre a sustentabilidade da dívida a médio prazo - mesmo levando em consideração o fato de que parte substancial do aumento de gastos públicos ao longo de 2009 referiu-se a gastos permanentes, e não a despesas anticíclicas propriamente ditas. É importante, contudo, que as autoridades avaliem com cuidado cada vez maior os custos e benefícios de suas políticas de gastos e transferências, sob pena de comprometerem o equilíbrio orçamentário e, consequentemente, perderem a capacidade de afetar o nível de atividade através da política fiscal.

\section{REFERÊNCIAS}

BLANCHARD, O.; PEROTTI, R. An empirical characterization of the dynamic effects of changes in government spending and taxes on output. Quarterly Journal of Economics, Cambridge, Massachusetts, v. 117, n. 4, p. 1329-1368, ago. 2002. 
ADAMS, F. G.; KLEIN, L. R. Performance of quarterly econometric models of the United States: a new round of model comparisons. In: KLEIN, L. R. Comparative performance of U.S. econometric models. Oxford University Press, 1991.

BURNSIDE, C.; EICHENBAUM, M.; FISCHER, J. D. M. Fiscal shocks and their consequences. Journal of Economic Theory, Nova Iorque, v. 115, p. 89-117, 2004.

CAVAlCANTI, M. A. F. H.; SILVA, N. L. C. Dívida pública, política fiscal e nível de atividade: uma abordagem VAR para o Brasil no período 1995-2008. In: XXXI Encontro Brasileiro de Econometria, 2009.

COGAN, J. F.; CWIK, T.; TAYLOR, J. B.; WIELAND, V. New Keynesian versus Old Keynesian government spending multipliers. NBER Working Paper, Cambridge, Massachusetts, n. 14782, Mar. 2009. Discussão.

EDELBERG, W. EICHENBAUM, M.; FISCHER, J. D. M. Understanding the effects of a shock to government purchases. Review of Economics Dynamics, p. 166-206, 1999.

FATÁS, A.; MIHOV, I. The effects of fiscal policy on consumption and employment. 2001. Mimeo.

FAVERO, C.; GIAVAZZI, F. Debt and the effects of fiscal policy. Federal Reserve of Boston Working Paper, n. 07-4, 2007. Discussão.

FERREIRA, P. C. Infra-estrutura pública, produtividade e crescimento. Pesquisa e Planejamento Econômico, v. 24, n. 2, p. 187-202, 1994.

FERREIRA, P. C. Investimento em infra-estrutura no Brasil: fatos estilizados e relações de longo prazo. Pesquisa e Planejamento Econômico, v. 24, n. 2, p. 187-202, 1994.

FORNI, L.; MONTEFORTE, L.; SESSA, L. The general equilibrium effects of fiscal policy. Estimates for the Euro Area. Journal of Public Economics, v. 26, n. 2, p. 231-252, 2009.

GIAVAZZI, F.; PAGANO, M. Can severe fiscal contractions be expansionary? Tales of two small European contries. NBER Macroeconomics Annual 1990, MIT Press, p. 75-111, 1990.

GIAVAZZI, F.; JAPPELLI, T.; PAGANO, M. Searching for non-linear effects of fiscal policy. Evidence from industrial and developing countries. European Economic Review, v. 44, n. 77, p. 1259-1289, 2000.

HEMMING, R.; KELL, M.; MAHFOUZ, S. The effectiveness of fiscal policy in stimulating economic activity: a review of the literature. IMF Working Paper, 02-208, 2002. Discussão.

ILZETZKI, E.; MENDOZA, E. G.; VEGH, C. A. How big are fiscal multipliers? Policy Insights, Centre for Economic Policy Research, n. 39, 2009. Discussão.

MENDONÇA, M. J. C.; MEDRANO, L. A.; SACHSIDA, A. Avaliando os efeitos da política fiscal no Brasil: resultados de um procedimento de identificação agnóstica. Texto para Discussão IPEA, n. 1377.

ORGANISATION FOR ECONOMIC COOPERATION AND DEVELOPMENT. OECD Economic Outlook Interim Report, March, 2009.

PERES, M. A. Os efeitos dinâmicos da política fiscal sobre a atividade econômica: um estudo para o caso brasileiro. Dissertação (Mestrado em Economia) - Departamento de Economia, Universidade de Brasília. 
PEROTTI, R. Estimating the effects of fiscal policy in OECD countries. CEPR Discussion Paper, n. 4842, 2005.

PEROTTI, R. In search of the transmission mechanism of fiscal policy. NBER Working Paper, n. 13143, 2007.

PIRES, M. C. C. Multiplicadores fiscais no Brasil: uma contribuição ao debate sobre políticas fiscais anticíclicas. In: XXXVII ENCONTRO NACIONAL DE ECONOMIA, ANPEC, 2009.

RAMEY, V. A.; SHAPIRO, M. D. Costly capital reallocation and the effects of government spending. Carnegie Rochester Conference on Public Policy, p. 145-194, 1998.

REIS, E. J.; CAVALCANTI, M. A. F. H.; CASTRO, A.S.; ROSSI JÚNIOR, J. L.; ARAÚJO, E. R.; HERNANDEZ, B. M. Model for projections and simulations of the Brazilian economy. Texto para Discussão IPEA, n. 619, 1999.

ROMER, C.; ROMER, D. The macroeconomic effects of tax changes: estimates based on a new measure of fiscal shocks. NBER Working Paper, n. 13264, 2007.

SILVA, A. M. A.; CÂNDIDO JÚNIOR, J. O. Impactos macroeconômicos dos gastos públicos na América Latina. Texto para Discussão IPEA, n. 1434, 2009.

UHLIG, H.; MOUNTFORD, A. What are the effects of fiscal policy shock? SFB 649 Discussion Paper, n. 039, 2005.

SPILIMBERGO, A.; SYMANSKY, S.; SCHINDLER, M. Fiscal multipliers. IMF Staff Position Note, n. 09/11, 2009.

VAN BRUSSELEN, P. Fiscal stabilization plans and the outlook for the world economy. NIME Policy Brief, Belgian Federal Planning Bureau, n. 01-2009, 2009. 
